\title{
ESTUDIO SOBRE EL MODELADO DEL CHORRO DE INYECCIÓN EN MOTORES DE COMBUSTIÓN INTERNA DIESEL CON APLICACIONES DIDÁCTICAS
}

\section{STUDY ON THE MODELING OF THE INJECTION JET IN DIESEL INTERNAL COMBUSTION ENGINES WITH DIDACTIC APPLICATIONS}

\section{Mawency Vergel ${ }^{11}$}

\section{Jhan Piero Rojas ${ }^{22}$}

\section{"Sofia Orjuela ${ }^{33}$}

Universidad Francisco de Paula Santander, Cúcuta, Colombia

\section{RESUMEN}

En este trabajo se estudia aborda los métodos y diseños experimentales didácticos -

1 Departamento de Matemáticas y Estadística, Universidad Francisco de Paula Santander, Norte de Santander, Cúcuta, Colombia, correo: mawencyvergel@ufps.edu.co Orcid: https://orcid.org/0000-0001-8285-2968

$2 \quad$ Facultad de Ingenierías, Universidad Francisco de Paula Santander, Cúcuta, Colombia. Correo jhanpierorojas@ufps.edu.co ORCID iD: https://orcid.org/0000-00032682-9880

investigativos usados para modelar el chorro líquido en motores de combustión interna Diésel y la validación de dichos modelos; además, busca conocer la tendencia actual en este campo. También, conocer los principios físicos y la tendencia tecnológica del proceso de inyección, con el fin de cimentar una base para futuros trabajos sobre el tema. Este acercamiento encontró que la tendencia actual es la de mejorar la combustión para producir 
menor materia contaminante, para así poder cumplir con las normativas vigentes, además se demostró que el modelado CFD es la herramienta más usada por los investigadores en el último año, debido a su relativo bajo costo y su precisión, en términos tecnológicos la inyección directa sigue siendo la opción más investigada y la cavitación presenta ahora un campo relativamente virgen de investigación debido a su complejidad, estas razones hacen a la cavitación un campo interesante de investigación, por otra parte el uso de bancos de inyección es la forma de validación más usada, debido a la facilidad para observar el proceso y controlar los distintos parámetros que gobiernan a la inyección, por otro lado los métodos ópticos son una opción interesantes para recoger datos debido a su exactitud.

PALABRAS CLAVE: Diésel, inyección, modelado, diseño experimental, principios físicos.

\section{INTRODUCCIÓN}

Actualmente, las exigentes normas de control de emisiones emitidas por los países han impulsado desarrollo de estrategias orientadas a su reducción. Dado que una combustión óptima no solo reduce los niveles de emisiones producidos por el motor, sino que también puede llegar a aumentar la potencia y reducir el consumo de combustible, el proceso de inyección ha pasado a tener un rol protagónico en el campo de la investigación. Sin embargo, la naturaleza no estacionaria de la combustión del chorro diésel, que involucra muchos procesos físicos y químicos complejos tales como la atomización, la evaporación, la cavitación y diversas reacciones químicas en ambientes altamente no homogéneos, hacen difícil y costoso el estudio de este fenómeno por los métodos habituales. Motivo por el cual, una técnica que reduzca los costos de experimentación para la investigación en esta área resulta de vital importancia.
La tendencia actual es la integración del modelamiento matemático a los procesos experimentales convencionales para estudio en asignaturas como motores térmicos o mecánicas de fluidos, con el fin de predecir el fenómeno de inyección, delimitando la función de la experimentación a un papel de validación, lo que reduce los costos y los tiempos de investigación requeridos drásticamente. Esto ha llevado a que en la última década surjan una variedad de estudios, que se tratará a lo largo del documento, que modelan la mezcla del chorro diesen en la cámara, su combustión y la posterior fase de emisión, algunos optan por modelar el fenómeno mediante sistemas empíricos o semi-empíricos (modelos cero-dimensionales, fenomenológicos y uní-dimensionales 1D), otros en cambio optan por las ecuaciones de conservación de masa y energía además de otros sistemas de ecuaciones, utilizando para su resolución la Dinámica de Fluidos Computacional (CFD) o hay quienes optan por la experimentación, creando hipótesis y demostrándolas por medio de experimentos.

Tanto para los experimentos como para el modelado es necesario conocer a profundidad los parámetros que influyen en la inyección con el fin de seleccionar solo los más relevantes y de esta forma economizar recursos y además afinar la precisión de los mencionados, para este fin es necesario abordar los principios físicos que rodean a la inyección.

A continuación, se expondrán los principios físicos que rodean al proceso de inyección y los principales hallazgos, esto es de suma importancia para el entendimiento de la didáctica en este tipo de estudios.

\section{PARÁMETROS EN ESTUDIOS DIDÁCTICOS DE SISTEMA DE INYECCIÓN DIESEL}

\subsection{VISCOSIDAD Y DENSIDAD DEL COMBUSTIBLE}


La viscosidad puede ser definida como la medida de la resistencia de un líquido a fluir, una alta viscosidad puede conllevar a problemas en el manejo del combustible por parte de las bombas y el sistema de inyección; además con diferencias de presiones menores a $85 \mathrm{MPa}$, el coeficiente de descarga disminuye con el aumento de la viscosidad del diésel, puede llegar a aumentar hasta un $10 \%$ con un aumento de la viscosidad de solo $6.4 \mathrm{~mm}^{2} / \mathrm{s}$ y un aumento de la diferencia de presión igual a $25 \mathrm{MPa}$, por otra parte el flujo másico se ve también afectado por la densidad, pudiendo aumentar un $13 \%$ con una disminución del $28 \%$ de la densidad, esta última no se ve afectada por la diferencia de Choi et al. (2013). Otro aspecto de la inyección que se ve afectado por la densidad es el tamaño de la gota, se ha demostró que al disminuir la densidad del diésel el chorro adquiere mayor penetración y ángulo de apertura, pero disminuyendo la calidad de la atomización debido a las fuerzas de Van der Waals.

El ángulo de apertura del chorro diésel también se ve afectado por la viscosidad y la densidad del gasoil, a medida que se aumenta la viscosidad, el ángulo de apertura es menor; mientras que con la densidad sucede lo contrario, a medida que la densidad aumenta el ángulo de apertura también aumenta, cabe recalcar que ninguna de estas relaciones es de exacta proporcionalidad, siendo estos dos aspectos más significativos cuando la tobera tiene una relación L/D menor a la unidad (Reitz and Bracco, 1982).

\subsection{PRESIÓN DE INYECCIÓN}

La presión del diésel al momento de ser inyectado dentro de la cámara de combustión tiene un gran efecto sobre los diferentes parámetros que gobiernan la combustión, entre ellos, hallamos el efecto que tiene sobre las emisiones, la inyección a altas presiones reduce la emisión de materia particulada (PM) sin aumentar las emisiones de NOx. Por otro lado, una mayor presión de inyección se traduce en un diámetro
Sauter reducido, mayor penetración de chorro, mayor flujo másico (Chen, 2013).

La presión inyección también tiene un efecto relevante sobre la mezcla entre el diésel y el aire. Está claro que un tamaño de gota menor favorece a la vaporización, resultando en una combustión más rápida (Chen, 2013). Cabe aclarar que una combustión rápida es necesaria para una combustión completa en los pequeños espacios de tiempo que se manejan a altas revoluciones dentro de la cámara de combustión, a altas presiones de inyección disminuye el tiempo de movimiento de la aguja, lo que favorece a una combustión más acelerada (Wang, 2010), Esta mezcla acelerada en adición con la mezcla de mejor calidad resulta en una menor emisión de humos y CO (Zhang, 2012).

\subsection{CAVITACIÓN}

El fenómeno de la cavitación, se define de manera sencilla como la formación de burbujas en un líquido, puede tener efectos negativos en una sistema hidráulico, estas burbujas pueden ser generadas por el la diferencia de velocidad entre el líquido que fluye en contacto con las paredes de las tuberías y el resto del flujo, esta diferencia de velocidades se traduce en una diferencia de presiones, creando vacíos y recirculación, si la presión estática del fluido llega a un valor por debajo de la presión de vapor de este, se produce una vaporización parcial del combustible, estas burbujas pueden ser comprimidas e implosionar a nivel molecular, removiendo material interno dañando el funcionamiento correcto del inyector. Por otra parte, la cavitación puede aumentar la velocidad de inyección y favorecer la atomización debido a la disminución de la viscosidad causada por la presencia de vapor (Payri, 2012).

Cuando se presenta la cavitación, el flujo másico colapsa y permanece constante al aumentar la presión de inyección (Payri, 2008). Esta característica se puede usar para determinar 
la presencia de la cavitación como método no intrusivo, además para prevenir este fenómeno se pueden usar toberas cónicas debido a la aceleración gradual que se presenta y el cambio menos abrupto del flujo (Payri, 2005). Incluso en recientemente estudios se ha hallado que un aumento del ángulo del cono de pulverización retrasa el comienzo de la cavitación (Payri, 2006). Aun no comprendemos a profundidad la influencia de este parámetro debido a su naturaleza no-estática y microscópica.

\subsection{ATOMIZACIÓN Y COEFICIENTE DE DESCARGA}

Cuando el diésel es inyectado esta toma forma de cono desde la salida de la tobera, este tipo de comportamiento es llamado régimen de ruptura de atomización y produce gotas de mucho menor diámetro que la tobera (Payri, 2006). Una correcta atomización genera gotas de tamaño reducido lo que ayuda a una combustión más completa y más eficiente con menor formación de contaminantes, se han determinado un modelo para definir el desarrollo de la atomización mediante el modelo hibrido KH-RT (Heywood, 2018).

El modelo $\mathrm{KH}-\mathrm{RT}$, explica que la atomización se realiza en 2 etapas. La primera de ellas la etapa $A$, consiste en una separación de la gota del chorro principal debido a la inestabilidad $\mathrm{KH}$, una vez se separó la gota, esta se somete a una ruptura secundaria debido a las inestabilidades entre KH Y RT (Beale, 1999). Para aplicar este modelo se deben conocer muchos parámetros entre ellas el ángulo del cono, la masa de pulverización inyectada y la velocidad del chorro inicial, y la longitud $L$ de ruptura líquida se calcula utilizando las ecuaciones introducidas por Lee (2002).

Por otra parte, han sido reportados estudios donde se muestra una relación entre la forma de la tobera de inyección y el desarrollo de la atomización (Badock, 1999). Por ejemplo, se ha determinado que cuando la relación L/D de la tobera es pequeña $(L / D<1.8)$ se desarrolla un área de pulverización amplia y esto puede conducir en tamaño de gota menor (Suh, 2008).

El coeficiente de descarga puede definirse como la razón adimensional que describe las pérdidas del flujo de combustible inyectado por el inyector, lo que quiere decir que es una medida de la eficiencia del flujo. Este se ve afectado tanto por la cavitación, densidad, viscosidad, geometría de la tobera y levantamiento de la aguja (Heywood, 2018).

\subsection{PENETRACIÓN Y ÁNGULO DE CHORRO}

La velocidad y la distancia a la que el chorro logra penetrar en la cámara de combustión tiene gran influencia sobre la mezcla diésel-aire, una sobrepenetración puede resultar en el choque con superficies frías (paredes de la camisa) que resultan en una combustión insuficiente aumentando las emisiones, y una penetración pobre resulta en una mezcla diésel-aire deficiente ya que una porción del aire no entra en contacto con el diésel. Se han creado diferentes correlaciones entre los experimentos y teoría de chorro gaseoso para predecir la penetración del chorro en la cámara de combustión (Hiroyasu, 1985).

Se puede definir el ángulo de chorro como el ángulo contenido por dos líneas rectas extraídas de la tobera de descarga para cortar los contornos de pulverización a una distancia específica de la cara del atomizador. Este ángulo se ve influenciado principalmente por el diámetro, la longitud de la tobera de inyección, el coeficiente de descarga, la viscosidad y la densidad (Rizk, 1987).

\subsection{SISTEMAS DE INYECCIÓN EN BANCOS DIDÁCTICOS}

El sistema de inyección es el responsable de entregar el combustible con las condiciones ideales dentro de la cámara de combustión, 
para ello tiene que cumplir con las siguientes funciones básicas:

- Introducir el combustible en las cantidades y presiones precisas en sincronía con el movimiento del cigüeñal.

- Atomizar el combustible para aumentar el área de contacto con el aire caliente y de esta forma acelerar la evaporación.

- Favorecer una mezcla homogénea entre el diésel y el aire que se encuentra dentro de la cámara de combustión.

Además, existen varias formas de regulación: mecánica, electrónicamente y electromecánicamente. Las mecánicas son las más antiguas y las que más problemas da al momento de poner a punto, pero es la más económica de las tres, luego se halla la electromecánica y electrónica, la primera de estas es más fácil de poner a punto que la segunda, pero la electrónica crea una independencia entre las revoluciones y la inyección, lo que puede favorecer al retraso de la inyección.

Dependiendo del modo en que se produce la inyección del combustible se pueden diferenciar los sistemas de inyección en dos grupos: Inyección indirecta o de inyección directa. La principal diferencia radica en la presencia de una pre cámara donde se inyecta el diésel y el aire (en el caso de la inyección indirecta). La inyección indirecta favorece la mezcla homogénea y reduce los requerimientos de calidad y de presión del chorro, el principal inconveniente de la inyección indirecta es la perdida de energía calórica producida por el aumento de la relación área/volumen, por lo que la tendencia industrial actual es hacia la inyección directa, en esta se inyecta el combustible directamente dentro de la cámara, no hay perdida de energía como en la inyección indirecta, pero el tiempo de mezcla se reduce drásticamente, aumentando las emisiones pero reduciendo el consumo de gasoil (Zhao, 1999). Incluso en los últimos años se han desarrollado estudios que contemplan una combinación de estos dos sistemas, llamados "dual-injection system", que consiste en la instalación de dos inyectores, uno dentro de la cámara de combustión y otro en el canal de admisión, este sistema reduce las emisiones emitidas por el motor (Wang, 2018).

Como ya se ha mencionado, los motores de encendido por compresión (MEC) son necesarios altos valores de presión en la inyección para lograr una atomización y una mezcla de la mejor calidad posible, sobre todo en los motores con sistema de inyección directa, por ello la principal diferencia entre los sistemas de inyección han sido la forma como se entregan estos valores de presiones. Se han ideado gran cantidad de sistemas de inyección, que podemos agrupar en dos grandes grupos: Sistemas de acción directa y sistemas de acumulación.

\section{SISTEMA DE INYECCIÓN BOMBA LINEAL}

El primer sistema de inyección desarrollado fue el sistema de inyección en línea, este tipo de bomba ideada por Robert Bosch a principios del siglo XX ha sido la más utilizada por no decir la única que funcionaba sobre todo en vehículos pesados, incluso se usó en turismos hasta la década de los 60 , pero se vio sustituida por las bombas rotativas más pequeñas y más aptas para motores rápidos. Este tipo de bombas es de constitución muy robusta y de una fiabilidad mecánica contrastada, por sus inconvenientes de tamaño, peso y que están limitadas a un número de revoluciones, esto las hace aptas para vehículos pesados, pero no para turismos.

Consiste en un pistón accionado por un árbol de levas, el diámetro del pistón determina la cantidad máxima de combustible que se puede inyectar, aunque la cantidad de combustible a inyectar puede ser cambiado gracias a unos 
canales de alivio mecanizados a los lados del pistón, los cuales redistribuyen más o menos diésel dependiendo del accionar de una corredera lateral.

\section{SISTEMA DE INYECCIÓN DE BOMBA ROTATIVA}

El sistema de inyección por bomba lineal tenía principalmente dos inconvenientes, primero su gran tamaño y segundo un costo elevado debido al requerimiento de una bomba por cada cilindro, para solucionar estos defectos se ideo el sistema de inyección de bomba rotativa que es mucho más compacta y además es lubricada por el mismo combustible que bombea, por lo que puede trabajar en cualquier posición.

El sistema de inyección de bomba rotativa consiste principalmente un distribuidor cilíndrico que va girando suministrando el combustible al inyector correspondiente al ir descubriendo el orificio de salida del combustible, la principal diferencia entre el sistema de inyección lineal y el rotativo, es que, aunque la compresión del combustible se sigue haciendo por medio de pistones, la dosificación no es echa por estos elementos.

\section{RESULTADOS DEL ESTUDIO PEDAGOGICO DE SISTEMAS DE INYECCIÓN}

\subsection{MODELADO Y SIMULACIÓN GENERAL}

El modelado a través de análisis computacional ha sido una mejora tecnológica que permite realizar estudios sin la necesidad de realizar largas experimentaciones físicas para poder entender el comportamiento y predecir el fenómeno de estudio, dando una gran ventaja en la parte económica y de tiempos de trabajo. De esta forma el estudio de los sistemas de inyección no se ha quedado atrás a la hora de utilizar las herramientas de modelado y simulación. El modelado de motores de combustión puede ser clasificado en dos grupos, los modelos termodinámicos que basados en la primera ley de la termodinámica el balance de masa y de energía y los modelos fluido-dinámicos basados en las ecuaciones que afectan el flujo. Por otro lado, Bracco (1975) clasifica los modelos en: cero-dimensional, cuasi-dimensional o fenomenológico y multidimensional, los límites para clasificar el tipo de modelo no están definidos entre dimensionales, termodinámicos y fluido-dinámicos, un modelo puede ser cero dimensional y termodinámico a la vez.

Para que un modelado, independientemente del tipo, sea exitoso y confiable, se deben de tener en cuenta ciertos criterios.

- Conocer el sistema a modelar: funcionamiento del sistema, variables de entrada, variables de salida.

- Conocimiento de los fenómenos físicos que afectan el sistema: propiedades físicas del fluido, cavitación, fricción.

- Dominio de las ecuaciones que rigen el sistema.

\subsection{MODELADO CERO DIMENSIONALES (0-D)}

El modelado cero dimensional se basa principalmente en la conservación de la energía, donde las variables implicadas en el proceso se consideran homogéneas en todo el espacio. Un modelo termodinámico cero-dimensional es eficiente para calcular aspectos de una forma global, por su versatilidad y baja complejidad (Payri, 2011). Los modelados cero-D han sido frecuentemente utilizados para diferentes aspectos en el proceso de inyección. Un ejemplo es el trabajo de Payri (2016) que realiza un estudio 0-D para determinar la influencia de la presión en el comienzo del rail y el flujo másico en un sistema common rail. Asumiendo un inyector en función de ciertos parámetros geométricos. Por otra parte, Soriano (2018) realiza un estudio para determinar la tasa de 
inyección de combustible con inyectores de solenoide, conociendo la presión de inyección, el tiempo de activación de la inyección, la temperatura de inyección y características de la boquilla del inyector, el modelo que realizo, es capaz de realizar la inyección única y la inyección dividida, es decir, la inyección y la preinyección.

\subsection{MODELADO CUASI-DIMENSIONALES O FENOMENOLÓGICO}

Los modelos fenomenológicos son básicamente modelos 0-D que incluyen alguna característica geométrica o detalles espaciales para alguna variable que rige el fenómeno, en comparación con los modelos multidimensionales, son más sencillos de ejecutar, pero requiere ajustar coeficientes con datos experimentales debido a las simplificaciones de este. Los modelos fenológicos se dividen en dos, los que modelan el chorro como un fluido continuo semiestacionario y unidimensional y los que dividen al chorro por paquetes. La diferencia entre estos es que el primero toma el chorro como un fluido continuo y no tiene en consideración los fenómenos de atomización y evaporación que ocurren alrededor de él, todo lo contrario, con el de paquetes que considera la mezcla entre aire y combustible gaseoso.

Los modelos fenomenológicos pueden estudiar cómo se desarrolla la posición y el tamaño al mismo tiempo. Algunos de los primeros modelos realizados fueron los desarrollados con $\mathrm{W}$. Austen (1960), que plantearon una relación entre la tasa de inyección del combustible y el calor liberado. Además de predecir las perdidas por transferencia de calor, velocidad de inyección y el retardo de la ignición (Sindhu, 2018).

\subsection{MODELADO MULTIDIMENSIONAL O CFD}

Los modelos multidimensionales son los más complejos de los tres mencionados debido a su capacidad de describir individualmente cada uno de los fenómenos que influyen en el proceso de inyección, los resultados del modelado CFD del flujo en el sistema de inyección detalla el proceso del flujo y su influencia en el rendimiento del motor, al comparar los resultados de la liberación de calor y la presión dentro del cilindro con datos experimentales se logra evidenciar que están muy cercanos dando una alta fiabilidad de los mismos (Reitz, 1995). La técnica de modelado CFD combinado con un modelo RAMS y usando el Numero de Cetano Derivado, muestra que la variabilidad de la reactividad química en lugar de las propiedades físicas rige en el tiempo de encendido.

\subsection{MODELADO LES (LARGE EDDY SIMULATION)}

EI modelo LES está basado en la filtración de ecuaciones (eliminar rangos de pequeñas escalas de soluciones de las ecuaciones de Navier-Stokes), este modelo simula flujos turbulentos resolviendo numéricamente las ecuaciones de Navier-Stokes, ignorando las escalas de longitud más baja y facilitando los costos computacionales, convirtiéndolo en un modelo económico y práctico para simular flujos turbulentos en situaciones geométricamente complejas, dándole una ventaja frente a el modelado DNS (Zhiyin, 2015).

Los modelos LES son de gran importancia para la simulación de los fenómenos complejos presentes en la formación y penetración del chorro de combustible (pulverización y turbulencia), aunque se han encontrado faltas de precisión en la pulverización del chorro y la distancia liquida en estudios sin combustión, principalmente relacionado con la configuración de los submodelos y el tamaño de la malla. Con la obtención de los submodelos adecuados y el nivel correcto de refinamiento de la malla, los resultados del modelo mejoran significativamente (Hindi, 2018). 


\subsection{MODELO SIMULACIÓN NUMÉRICA DIRECTA (DNS)}

El modelo DNS es el más preciso para simular flujos turbulentos, debido a que resuelve las ecuaciones de Navier-Stokes numéricamente usando una malla muy fina para poder resolver todas las escalas presente en el fluido, desde los remolinos más grandes hasta los más pequeños, esta precisión en los cálculos consume muchos recursos computacionales haciendo que este tipo de modelado sea muy costos y de gran dificultad para aplicarlo en geometrías complejas; por esta razón el modelo DNS es más usado para modelar el chorro en el proceso de combustión (Krisman, 2016).

Una vez definidos los modelos, hay que aclarar las técnicas usadas para su validación, un modelo sin validación no tiene sentido, hay que corroborar la interpretación del fenómeno por parte del modelo, si es correcta y que tan exacta es, de esta forma podemos usar los resultados por parte del modelo con seguridad.

\subsection{MODELADO LES (LARGE EDDY SIMULATION)}

En el ítem anterior se mostraron los diferentes tipos de modelos computacionales para conocer y predecir los fenómenos que ocurren en el proceso de inyección, pero cada modelo debe ser verificado a través de la comparación de los resultados arrojados del modelo con datos reales, con el fin de conocer la valides y exactitud de los resultados obtenidos por el modelo. Comparar los datos de un modelo con un motor en funcionamiento puede llegar a ser muy complejo y costoso debido a la dificultad para tomar las mediciones. Por esta razón se han creado modelos de experimentación que facilitan la toma de mediciones y reducen los costos de la experimentación, a continuación, se enumeraran los principales tipos de validación.
Las maquetas de inyección son bancos experimentales que busca replicar las condiciones en las que se encontraría un chorro diésel al momento de ser inyectado, las maquetas deben de replicar las condiciones de presión, temperatura y densidad, tanto del combustible como la del aire, además de permitir la observación y toma de datos de una forma fácil, retirar el combustible inyectado y permitir el uso de diferentes sistemas de inyección. Un ejemplo es el banco experimental usado por Simón Marčič (2018), en donde para cada parámetro termodinámico se puede realizar un ajuste independiente, las presiones que maneja son dependientes del sistema de inyección y tiene una ventana que posibilita una fácil visibilidad del proceso. Estas condiciones le proporcionan una mejor comprensión de las variables que afectan en el estudio.

\subsection{TÉCNICAS LASER Y ÓPTICAS}

Para la toma y adquisición de datos en una maqueta de inyección experimental, es importante que la técnica de medición a usar no interfiera con el desarrollo del chorro, la anemometría de fase doopler es capaz de determinar la velocidad y el tamaño de la gota sin afectar el desarrollo del chorro, la técnica se basada en la luz relejada por la gota que pasa a través de un volumen de control definido por la intersección de dos haces de luz láser (Jiménez, 1998).

Otra técnica usada comúnmente es la toma de imágenes con cámaras de alta velocidad, esta técnica permite caracterizar el chorro de forma macroscópica, permitiendo obtener datos como la longitud del chorro, el ángulo de apertura del chorro, el volumen y la masa del chorro (Yu, 2017).

Por medio de cámaras validan los modelos usados para estudiar las características del chorro sometido a altas presiones y conocer 
el efecto de la temperatura en la cavitación en toberas de varios orificios.

El uso de rayos $x$ es otra técnica usada para el estudio de la inyección (Huang, 2017). Se usa esta técnica para estudiar el efecto de la posición de la aguja dentro del inyector y el número de orificios por inyector.

\subsection{DISCUSIÓN}

Anteriormente en el documento se afirmó que la tendencia actual es el modelado CFD, para justificar este argumento el equipo realizo una revisión de la bibliografía disponible. La metodología usada consistió básicamente en usar tres palabras claves (diesel, injection y modeling) para realizar una búsqueda por año en la plataforma sciencedirect, revisando los 20 primeros resultandos y clasificando los tipos de modelados usados en cada uno, los resultados de la búsqueda se muestran en las Figuras 1 y 2.

Figure 1: Tendencia de los diferentes tipos de estudios de inyección. Elaborado por los autores a partir de datos de plataforma Science Direct.

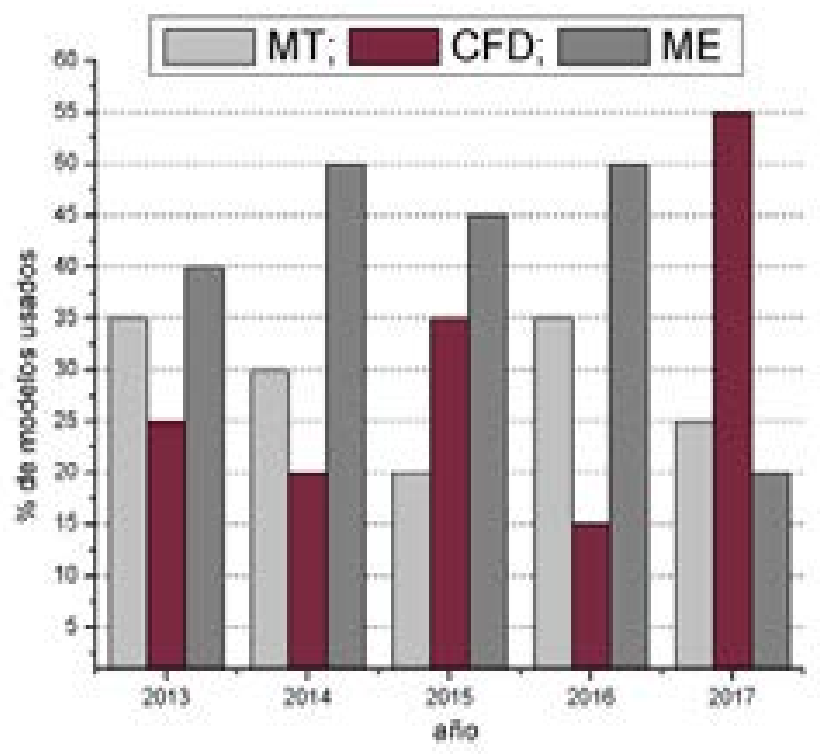

Figure 2: Tendencia del modelado CFD a través en los últimos años. Elaborado por los autores a partir de datos de plataforma Science

Direct.

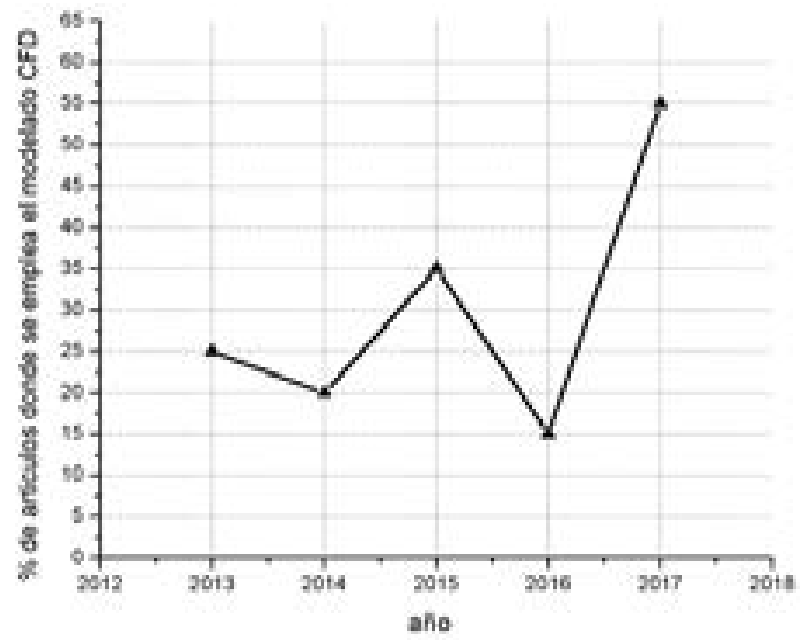

Se puede ver como el modelado CFD ha tomado fuerza a través de los años, pasando de ser usado por un $25 \%$ de los estudios en el 2013 , a ser usado en un poco más de la mitad de los estudios de la muestra en el 2017.

\section{CONCLUSIÓN}

En este trabajo se ha hecho un completo estudio de la bibliografía referente a los temas que rodean a la inyección enfocándose solamente en la inyección y no se implicó con la combustión del chorro, todo esto con el objetivo de cimentar bases para trabajos posteriores, a partir de esta revisión se extrajeron las siguientes conclusiones.

- De todos los fenómenos físicos que rodean a la inyección es la cavitación la que tiene el menor soporte empírico, esto sumado a que es un parámetro que afecta en gran medida a la inyección lo convierte en un objetivo de investigación interesante. 
- $\quad$ El chorro líquido, debido a su naturaleza no estática y su dificultad de medición es una región no tan investigada.

- La relativa novedad de combustibles alternativos como el biodiesel o los sistemas de multi-combustible, nos entrega un campo de investigación nuevo, amplio y muy interesante.

- La relativa novedad y escasa aplicación de los sistemas dual-inyección ha resultado en una escasa literatura detrás, dejando a este sistema de inyección como un campo interesante de investigación.

- El mejor método para modelar el chorro, teniendo en cuenta la relación recursos/ exactitud, es el modelado LES, para fluidos turbulentos.

Si se requiere mayor exactitud en un parámetro en específico la tendencia es combinar los modelos, con la mezcla de los modelos se puede profundizar en los parámetros que rigen el fenómeno manteniendo la economía de los estudios.

\section{REFERENCIAS BIBLIOGRÁFICAS}

Austen, A. E. W., \& Lyn, W. T. (1960). Relation between fuel injection and heat release in a direct-injection engine and the nature of the combustion processes. Proceedings of the Institution of Mechanical Engineers: Automobile Division, 14(1), 47-62.

Badock, C., Wirth, R., Fath, A., \& Leipertz, A. (1999). Investigation of cavitation in real size diesel injection nozzles. International journal of heat and fluid flow, 20(5), 538544.

Beale, J. C., \& Reitz, R. D. (1999). Modeling spray atomization with the Kelvin-Helmholtz/ Rayleigh-Taylor hybrid model. Atomization and sprays, 9(6).
Bracco, F. V. (1975). Introducing a new generation of more detailed and informative combustion models. SAE Transactions, 3317-3340.

Chen, P. C., Wang, W. C., Roberts, W. L., \& Fang, T. (2013). Spray and atomization of diesel fuel and its alternatives from a single-hole injector using a common rail fuel injection system. Fuel, 103, 850-861.

Choi, W. C., Yoo, I. S., Park, M. R., \& Chung, M. K. (2013). Experimental study on the effect of blade angle on regenerative pump performance. Proceedings of the Institution of Mechanical Engineers, Part A: Journal of Power and Energy, 227(5), 585-592.

Heywood, J. B. (2018). Internal combustion engine fundamentals. McGraw-Hill Education.

Hindi, G., Paladino, E. E., \& de Oliviera Jr, A. A. M. (2018). Effect of mesh refinement and model parameters on LES simulation of diesel sprays. International Journal of Heat and Fluid Flow, 71, 246-259.

Hiroyasu, H. (1985, September). Diesel engine combustion and its modeling. In Comodia (Vol. 85, pp. 53-75).

Huang, W., Moon, S., Gao, Y., Li, Z., \& Wang, J. (2017). Eccentric needle motion effect on near-nozzle dynamics of diesel spray. Fuel, 206, 409-419.

Jiménez, D. C. (1998). Estudio teóricoexperimental del chorro libre diesel isotermo (Doctoral dissertation, Universitat Politècnica de València).

Krisman, A., Hawkes, E. R., Talei, M., Bhagatwala, A., \& Chen, J. H. (2016). Characterisation of two-stage ignition in diesel enginerelevant thermochemical conditions using 
direct numerical simulation. Combustion and Flame, 172, 326-341.

Lee, C. S., \& Park, S. W. (2002). An experimental and numerical study on fuel atomization characteristics of high-pressure diesel injection sprays. Fuel, 81(18), 2417-2423.

Marčič, S., Marčič, M., Wensing, M., Vogel, T., \& Praunseis, Z. (2018). A simplified model for a diesel spray. Fuel, 222, 485-495.

Payri González, F., \& Desantes Fernández, J. M. (2011). Motores de combustión interna alternativos. Editorial Universitat politécnica de valencia.

Payri, R., Gimeno, J., Novella, R., \& Bracho, G. (2016). On the rate of injection modeling applied to direct injection compression ignition engines. International Journal of Engine Research, 17(10), 1015-1030.

Payri, F., Payri, R., Salvador, F. J., \& MartínezLópez, J. (2012). A contribution to the understanding of cavitation effects in Diesel injector nozzles through a combined experimental and computational investigation. Computers \& Fluids, 58, 88101.

Payri, R., Salvador, F. J., Gimeno, J., \& Zapata, L. D. (2008). Diesel nozzle geometry influence on spray liquid-phase fuel penetration in evaporative conditions. Fuel, 87(7), 1165-1176.

Payri, R., García, J. M., Salvador, F. J., \& Gimeno, J. (2005). Using spray momentum flux measurements to understand the influence of diesel nozzle geometry on spray characteristics. Fuel, 84(5), 551-561.

Payri, R., García, J. M., Salvador, F. J., \& Gimeno, J. (2006). Using spray momentum flux measurements to understand the influence of diesel nozzle geometry on spray characteristics. Fuel, 84(5), 551-561.

Reitz, R. D., \& Bracco, F. V. (1982). Mechanism of atomization of a liquid jet. The physics of Fluids, 25(10), 1730-1742.

Reitz, R. D., \& Rutland, C. J. (1995). Development and testing of diesel engine CFD models. Progress in Energy and Combustion Science, 21(2), 173-196.

Rizk, N. K., \& Lefebvre, A. H. (1987). Prediction of velocity coefficient and spray cone angle for simplex swirl atomizers. International Journal of Turbo \& Jet-Engines, 4(1-2), 6574.

Sindhu, R., Rao, G. A. P., \& Murthy, K. M. (2018). Effective reduction of NOx emissions from diesel engine using split injections. Alexandria Engineering Journal, 57(3), 1379-1392.

Soriano, J. A., Mata, C., Armas, O., \& Ávila, C. (2018). A zero-dimensional model to simulate injection rate from first generation common rail diesel injectors under thermodynamic diagnosis. Energy, 158, 845-858.

Suh, H. K., \& Lee, C. S. (2008). Effect of cavitation in nozzle orifice on the diesel fuel atomization characteristics. International journal of heat and fluid flow, 29(4), 10011009.

Wang, X., Huang, Z., Kuti, O. A., Zhang, W., \& Nishida, K. (2010). Experimental and analytical study on biodiesel and diesel spray characteristics under ultra-high injection pressure. International journal of heat and fluid flow, 31(4), 659-666.

Wang, Y., Yu, X., Ding, Y., Du, Y., Chen, Z., \& Zuo, X. (2018). Experimental comparative study on combustion and particle emission 
of $\mathrm{n}$-butanol and gasoline adopting different injection approaches in a spark engine equipped with dual-injection system. Fuel, 211, 837-849.

Yu, S., Yin, B., Jia, H., \& Yu, J. (2017). Numerical research on micro diesel spray characteristics under ultra-high injection pressure by Large Eddy Simulation (LES). International Journal of Heat and Fluid Flow, 64, 129-136.

Zhang, G., Qiao, X., Miao, X., Hong, J., \& Zheng, J. (2012). Effects of highly dispersed spray nozzle on fuel injection characteristics and emissions of heavy-duty diesel engine. Fuel, 102, 666-673.

Zhao, F., Lai, M. C., \& Harrington, D. L. (1999). Automotive spark-ignited direct-injection gasoline engines. Progress in energy and combustion science, 25(5), 437-562.

Zhiyin, Y. (2015). Large-eddy simulation: Past, present and the future. Chinese journal of Aeronautics, 28(1), 11-24. 